\title{
Identifying and supporting autistic preschoolers and their families
}

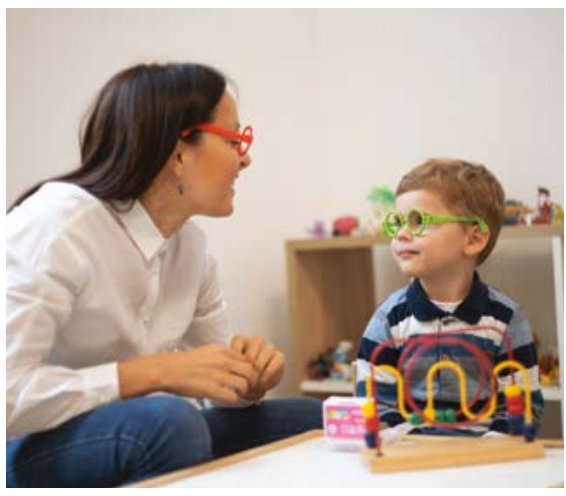

CPD

Amanda Brignell, Georgina Cox, Alexandra Ure, Charmaine Bernie, Katrina Williams

\section{Background}

In Australia, preschoolers are being identified and diagnosed as autistic.

\section{Objective}

The aim of this article is to describe the different paths preschool children and their families can take from identification of developmental or behavioural concerns to ongoing support, intervention and healthcare.

\section{Discussion}

There are many ways in which general practitioners, working alongside other professionals and with relevant services, can assist each child and family.
AUTISM is a developmental condition that affects social communication and behaviour. ${ }^{1}$ In Australia, significant progress has been made in the development of systems to detect autism early, ${ }^{2}$ with most children now diagnosed during the preschool years. ${ }^{3}$

Parents (a term used in this article to refer to all primary caregivers), child care educators and healthcare professionals are well placed to monitor the achievement of language, motor and social/emotional milestones in preschoolers. When development is not progressing as expected, as seen in autism, identifying differences early can assist in providing advice, interventions and supports to families, leading to positive outcomes. ${ }^{4-6}$

Children with developmental or behavioural differences may present to a general practitioner (GP) in various ways. When seeing a child who may have autism, it is beneficial to adopt a holistic assessment approach, with all diagnostic possibilities considered. Key assessment elements should include language, social emotional and cognitive development, as the basis for understanding ability and behavioural differences. Consideration of environmental stressors and family dynamics is also important, as both can affect child development and behaviour. Depending on the severity and type of difficulties, there is a range of healthcare professionals who can assess, intervene and support the child and family (Figure 1). The type of professionals involved and their setting (eg private, community health and hospital outpatient clinics) will vary according to local availability and parent resources. Advice, interventions and supports should be individually tailored to the child's and family's needs, including consideration of the wellbeing and support needs for parents and siblings. This approach will ensure best care, regardless of the diagnostic outcome. As discussed further in this article, GPs are integral to each step in a child and family's journey.

\section{Identification}

In Australia, GPs are often viewed as the 'gatekeepers' for care and are therefore key decision makers in identifying and referring children to services. ${ }^{7}$ When identifying children with neurodevelopmental differences, GPs are well placed to offer surveillance using standardised tools or to refer to Child Health Nurses (CHNs) for surveillance completion. Alternatively, GPs can be the first to hear of parents' concerns about their child or about concerns that have been raised by others, such as early years educators (eg preschool educators, day care staff), who are well placed to observe 
child behaviour in social settings and identify developmental and behavioural differences. ${ }^{8}$

The role of CHNs in development and behaviour varies across Australia. With no agreement regarding a universal population surveillance policy, there are different views regarding whether the benefits are sufficient to justify autism surveillance. ${ }^{9,10}$ As such, it is important that GPs are aware of their local surveillance services and how to access them when needed.

\section{Post-identification of neurodevelopmental or behavioural differences}

As shown in Figure 1, there are multiple professionals involved in assessment, intervention and support for children with developmental and behavioural concerns.

\section{Assessment}

Audiologists have a critical role in the early care pathway for autistic children. ${ }^{11}$ If language is delayed or a child is not responding to cues from their parents, hearing impairment must be excluded. Less commonly, visual acuity requires assessment if there are concerns about vision as a reason for poor eye contact and visual engagement. ${ }^{12}$ Ophthalmology referral is indicated for concerns about eye movements, strabismus, ptosis or a condition that has both vision and developmental difficulties.

If a child's developmental milestones are delayed or their behaviours are causing problems or are different than expected for their ability, referral for further assessment by allied health providers is indicated. In the first instance, this could be with a care provider most directly relevant to the differences, such as a speech pathologist for speech, language and social communication; an occupational therapist for functional skills or sensory-motor difficulties; or a psychologist for cognitive skills and early behavioural or emotional support. If there are concerns about the child's environment, then social work expertise will assist, and if there are risks to the child, mandatory reporting should be completed. Detailed assessment in the aforementioned domains can provide key information needed for identifying intervention targets and assessing whether a child's behaviour is in keeping with, or different to, expectations for their age

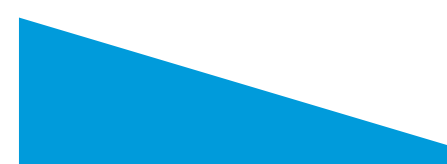

Identifying developmental

and behavioural differences

Either:

- surveillance is planned*

parents present with a

concern

- early childhood educators

identify differences in

development

- concerns are identified

when child presents for

other healthcare issue

GP and/or CHN completes

Tier $1^{\dagger}$ surveillance

- $\mathrm{CHN}$ completes Tier $2^{\ddagger}$

surveillance if concerns

identified at Tier $1^{\dagger}$
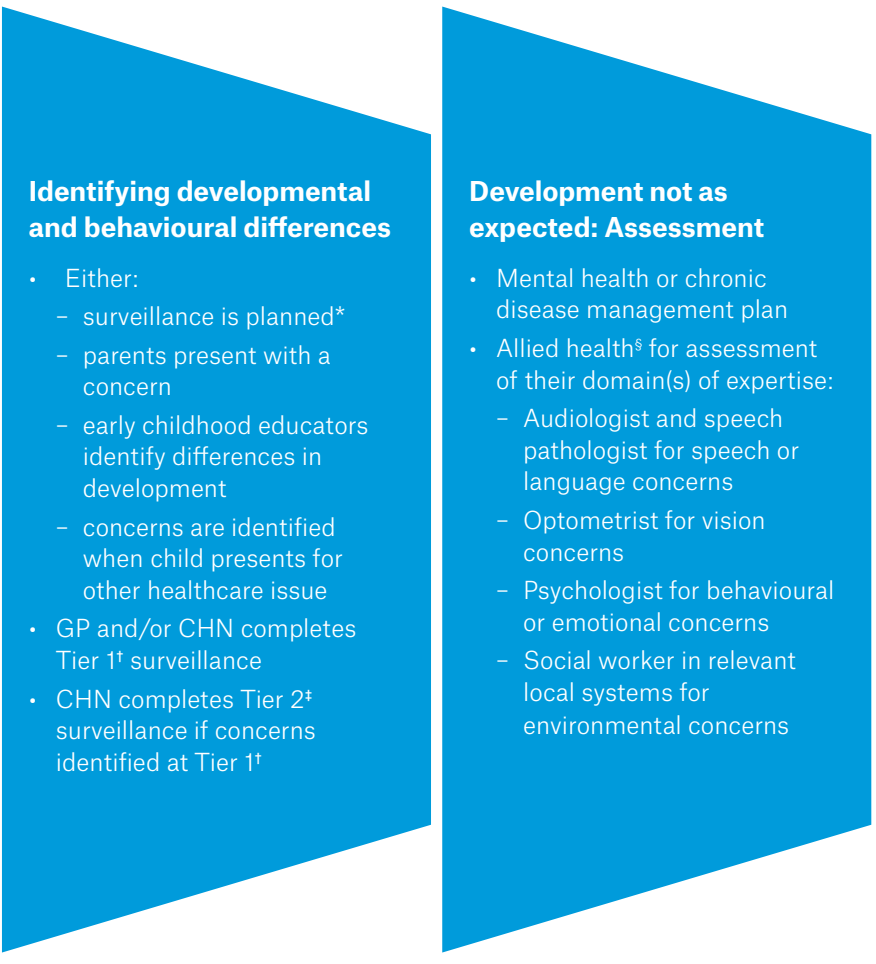

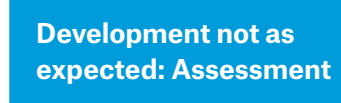

- Mental health or chronic

disease management plan

- Allied health ${ }^{\S}$ for assessment

of their domain(s) of expertise:

Audiologist and speech

pathologist for speech or

language concerns

Optometrist for vision

concerns

Psychologist for behavioural

or emotional concerns

Social worker in relevant

local systems for

environmental concerns

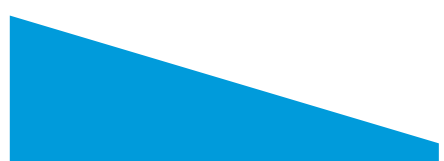

Development not as

expected: Intervention

and support

- Allied health interventions ${ }^{\S}$

Parent support to navigate

services and seek interventions

Support for parents to identify

goals

Psychology§ for:

- child behaviour

parents to manage behaviour

child or family mental health

NDIS for ECIS

Child care/preschool

Monitor progress
Figure 1. The different levels that can indicate general practitioner involvement

*As recommended in the relevant state or territory

tTier 1: population surveillance including children with no identified concerns

¥Tier 2: surveillance of children with concerns identified

sWorking in a community health, private or hospital outpatient setting

CHN, child health nurse; ECIS, Early Childhood Intervention Services; GP, general practitioner; NDIS, National Disability Insurance Scheme 
and ability. Such assessments can occur prior to a diagnostic assessment, as part of public community health services, a National Disability Insurance Scheme (NDIS) plan or in private with funding support from mental health or chronic disease care plans, if criteria are met.

\section{Intervention and support}

On the basis of assessment findings, allied health professionals can offer supports and interventions for the child and family (Figure 1). Allied health professionals can also assist families to access early childhood intervention from services funded by the National Disability Insurance Agency. ${ }^{3,13}$ There is no need to delay access to interventions and supports while waiting for a diagnostic assessment, as entry is based on functional difficulties. Early childhood educators, other day care providers and kindergarten or preschool teachers have opportunities to support child behaviour and are well placed to implement strategies in the child's social settings. ${ }^{8}$ Additional supports are also needed, such as assisting parents to identify their key goals, navigate services and seek interventions, alongside monitoring of a child's progress. Emerging evidence about parent coaching and stepped care provides guidance on how to implement structured and personalised care. ${ }^{14-17}$

It is important to recognise the stressful nature of the period in which a family begins to notice differences in development and to consider a potential diagnosis of autism for their child. ${ }^{17,18}$ Regardless of whether a swift and affirming response or a 'watch and wait' with active monitoring and follow-up is deemed most appropriate, there is an opportunity to support families to deal with uncertainties and fears. Where monitoring and follow-up is planned, knowing the details of the plan will be reassuring for the family. Interventions and supports tailored to the needs of parents and siblings should also be considered. If parents develop or have pre-existing mental health needs, then a separate referral to support them is required.

The following case provides an example of a child who required assessment of speech and language delays with some intervention, but where a full multidisciplinary team diagnostic assessment was not required.

\section{CASE 1: NASIR}

Nasir, aged two years and two months, attended his local general practice clinic because his mother was concerned that he was not talking and tended not to look at a person's face when making sounds. Nasir's mother had read about autism on the internet and was worried that Nasir was autistic. She wanted help as soon as possible but did not know which professional she should see.

Nasir's GP referred him to the $\mathrm{CHN}$ for surveillance. The results showed Nasir was behind in language but had met all his other milestones. The GP referred Nasir for a hearing assessment, which was normal. The GP also recommended child care and a speech pathology assessment to assess Nasir's communication and social skills. The speech pathologist identified an expressive language delay and saw Nasir for a short block of intervention, resulting in good progress. As his language improved, so did his eye contact, and he was discharged after four months. In this case, a referral to a paediatrician and other allied health services was not needed.

\section{Referral to a paediatrician and/or multidisciplinary assessment team}

Referrals to secondary and tertiary service providers may occur at parental request, in collaborative decision making between the parents and GP, or be instigated by the GP or other health professionals following identification of developmental or behavioural concerns. ${ }^{19}$ In Australia, there is a lack of standard information required for referral, and different services have different criteria and processes, ${ }^{15,20}$ which means that GPs need to be familiar with their local services and referral requirements. $^{21}$

Another common problem is extended waiting times for paediatric consultation or multidisciplinary assessments. This means that alternative strategies, as described for interventions in Figure 1, are needed to ensure a child and their family are receiving intervention and support while waiting.

When discussing indications for referral, it is preferable to discuss the child's abilities using strengths-based language, to ensure conversations are family-centred and to consider the broader sociocultural context.

\section{Paediatrician roles}

Paediatricians have an important role in symptom evaluation, case management and diagnosis. If a child is dysmorphic or there is a history of regression in development or neurological symptoms or signs, referral to a paediatrician is indicated. Exposures and complications during pregnancy (eg medication or substance use, gestational diabetes and hypertension) linked to differences in neurodevelopment should also trigger a paediatrician referral. ${ }^{22}$

Chromosomal microarray and fragile $\mathrm{X}$ testing is considered best practice for children presenting with developmental delay or intellectual disability, signs of autism and/or congenital abnormalities to investigate the possibility of genetic conditions. ${ }^{23,24}$ Examples of genetic conditions associated with autism include tuberous sclerosis and fragile $\mathrm{X}$ syndrome. ${ }^{25}$ When a child is diagnosed as autistic, referral to a genetic counsellor may be indicated so parents can be counselled regarding the recurrence risk for future children.

Paediatrician input for decisions about referral to a multidisciplinary clinic is also valuable and is required by some services. Paediatricians can also provide care coordination and long-term management for some children and their families.

\section{Diagnostic assessment}

If there are concerns regarding development or behaviour that involve multiple domains, then assessments by multiple disciplines are needed. In addition, it is important to consider the relevance of findings in relation to the child's environment, whether difficulties occur in multiple settings, and the impact that difficulties are having on activities 
and community participation..$^{21}$ There is no guidance for the minimum level or type of symptomatology to 'trigger' a referral for diagnostic assessment, but key factors for consideration would be significant differences from peers across more than one domain, a lack of response to community or privately accessed interventions, and family complexity.

A paediatrician or psychiatrist working in a private setting may commence a multidisciplinary assessment using Medicare Benefits Schedule items designed for that purpose. Alternatively, publicly funded hospital or communitybased multidisciplinary teams are now available in many parts of Australia, but local knowledge is required about their location and intake criteria. A clinical description of the way assessment, intervention and support can be offered to an autistic child is provided in Case 2.

\section{CASE 2: AMELIA}

Amelia's father first brought her to the family general practice clinic at the age of four years because of concerns about Amelia's lack of interest in people, use of only two-word phrases, dislike for loud noises and interest in horses to the exclusion of everything else. Amelia also had difficulties falling asleep and with day and night time wetting. The GP arranged for Amelia to have a hearing assessment (which was normal), referred Amelia to a paediatrician working in private practice and supported the family to apply for early childhood intervention from the NDIS. The paediatrician arranged genetic testing and referred Amelia to colleagues for speech and language and cognitive assessments. Amelia was diagnosed with an intellectual disability and autism, with no genetic cause identified.

For the following 18 months post-diagnosis, Amelia was supported by the NDIS with an early intervention program including speech therapy and positive behaviour support, with an initial focus on improving sleep and achieving toilet training. Amelia attended a child care setting, and her NDIS early intervention team worked with the child care staff and family to ensure consistency in visual schedules, use of visual aids and behaviour management.

Amelia made progress and was ready for school. Her family were supported by her NDIS team and child care professionals to approach mainstream and specialist schools and to make their selection. A paediatric review was also scheduled, where all assessment results were pulled together and further discussions about school placement and ongoing care provisions took place. A new report and recommendations were prepared for Amelia's family, school and the NDIS. This report and recommendations focused on both her strengths and difficulties to inform appropriate funding, education and care options for Amelia. This was also sent to the GP so that informed coordination of care and support could continue.

\section{Post-diagnosis}

Children diagnosed as autistic usually require some level of ongoing care coordination. This is more likely when there are associated medical conditions that require intervention and if activities and participation are reduced for the child. GPs are well placed to adopt care coordination, ${ }^{7,26}$ with capacity to assist with NDIS plans, mental healthcare plans for co-occurring conditions and behaviour management, and chronic disease management plans if these are not already in place (Figure 1). GPs can also manage medication with guidance from relevant specialists and can assist with linking families with local support services.

The autism assessment and diagnosis process can trigger a range of emotions for families, adding to prior stressors. ${ }^{27}$ Establishing positive relationships with parents allows for opportunities to monitor their wellbeing, provide diagnosis-related resources and offer referrals for individual and group-based supports as needed. ${ }^{28-30}$

\section{Conclusion}

GPs have an important role in identifying key developmental and behavioural differences; coordinating care through care planning; organising referrals to relevant services for further assessment, intervention and supports; and providing ongoing care for each child and their family. Knowledge of the family's resources and of local services will inform decision making regarding the most appropriate accessible services. Much can be done prior to a diagnosis to assist children and their families and to ensure children's needs are correctly identified so they are able to access appropriate services at the most beneficial time.

\section{Key points}

- Preschoolers may present with developmental and behavioural differences.

- Primary healthcare providers are critical for identification and preliminary assessment.

- Health assessments, including hearing and developmental domains, guide best care.

- Autistic children require ongoing support, intervention and healthcare.

\section{Authors}

Amanda Brignell BAppSci (Speech Pathology), MPH, PhD, Post-Doctoral Research Fellow, Department of Paediatrics, Monash University, Vic; Research Fellow, Murdoch Children's Research Institute, Vic; Senior Lecturer, Australian Catholic University, Vic Georgina Cox BSc (Hons), DClinPsych, PhD, Senior Research Fellow, Department of Paediatrics, Monash University, Vic; Clinical Psychologist, Department of Neurodevelopment and Disability, The Royal Children's Hospital, Vic

Alexandra Ure BBSc (Hons), M.Psych (clinical), PhD, Research Fellow, Department of Paediatrics, Monash University, Vic; Senior Clinical Psychologist, Monash Children's Hospital, The Royal Children's Hospital, Vic; Honorary Fellow, Murdoch Children's Research Institute, Vic

Charmaine Bernie BOccThy (Hons), Department of Paediatrics, University of Melbourne, Vic; Department of Allied Health, The Royal Children's Hospital, The Murdoch Children's Research Institute, Vic

Katrina Williams, MBBS, FRACP, FAFPHM, MSc, PhD, Professor and Head of Department of Paediatrics, Monash University Vic; Developmental Paediatrician and Director of Research, Monash Children's Hospital, Vic

Competing interests: $\mathrm{KW}$ declared personal fees from Exisle Publishing in the form of royalties for a book titled Understanding autism: An essential guide for parents, as well as consulting fees from the National Disability Insurance Scheme for advice regarding early childhood intervention for autism, paid to KW's institution.

Funding: None.

Provenance and peer review: Commissioned, externally peer reviewed. 


\section{Correspondence to:}

katrina.williams@monash.edu

\section{References}

1. American Psychiatric Association. Diagnostic and statistical manual of mental disorders. 5th edn. Arlington, VA: American Psychiatric Association, 2013.

2. Mozolic-Staunton B, Donelly M, Yoxall J, Barbaro J. Early detection for better outcomes: Universal developmental surveillance for autism across health and early childhood education settings. Res Autism Spectr Disord 2020;71:101496. doi: 10.1016/j.rasd.2019.101496.

3. May T, Williams K. Brief report: Gender and age of diagnosis time trends in children with autism using Australian Medicare data. J Autism Dev Disord 2018;48(12):4056-62. doi: 10.1007/s10803018-3609-7.

4. Orsmond Gl, Seltzer MM. Siblings of individuals with autism spectrum disorders across the life course. Ment Retard Dev Disabil Res Rev 2007;13(4):313-20. doi: 10.1002/mrdd.20171.

5. Perry A, Koudys J, Prichard A, Ho H. Follow-up study of youth who received $\mathrm{EIBI}$ as young children. Behav Modif 2019:43(2):181-201. doi: 10.1177/0145445517746916.

6. Elder JH, Kreider CM, Brasher SN, Ansell M. Clinical impact of early diagnosis of autism on the prognosis and parent-child relationships. Psychol Res Behav Manag 2017;10:283-92. doi: 10.2147/ PRBM.S117499.

7. Jeyendra A, Rajadurai J, Chanmugam J, et al. Australian general practitioners' perspectives on their role in well-child health care. BMC Fam Pract 2013;14:2. doi: 10.1186/1471-2296-14-2.

8. Jordan R, Roberts JM, Hume K. The SAGE handbook of autism and education. London, UK: SAGE Publications Ltd, 2019.

9. Barbaro J, Ridgway L, Dissanayake C. Developmental surveillance of infants and toddlers by maternal and child health nurses in an Australian community-based setting: Promoting the early identification of autism spectrum disorders. J Pediatr Nurs 2011;26(4):334-47. doi: 10.1016/j.pedn.2010.04.007.

10. Eapen V. Early identification of autism spectrum disorder: Do we need a paradigm shift? Aust N Z J Psychiatry 2016;50(8):718-20. doi: 10.1177/0004867416652733.

11. Brebner C, Lind C, Bickford J, Callahan L. Speech pathology and audiology: Assessment and intervention for communication impairment. In: Willis E, Reynolds L, Rudge T. Understanding the Australian Health Care System. 4th edn. Chatswood, NSW: Elsevier, 2019; p. 454-68.

12. Bakroon A, Lakshminarayanan V. Visual function in autism spectrum disorders: A critical review. Clin Exp Optom 2016;99(4):297-308. doi: 10.1111/ cxo.12383.

13. May T, Roberts J, Webber M, et al. Brief history and user's guide to the Australian National Disability Insurance Scheme. J Paediatr Child Health 2018;54(2):115-20. doi: 10.1111/jpc.13748.

14. Connolly M, Gersch I. A support group for parents of children on a waiting list for an assessment for autism spectrum disorder. Educ Psychol Pract 2013;29(3):293-308. doi: 10.1080/02667363.2013.841128.

15. Bernie C, Williams K, O'Connor B, Rogers S, May T. Referral, assessment and use of screening measures related to autism spectrum disorder at a tertiary hospital setting. J Autism Dev Disord 2020. doi: 10.1007/s10803-020-04725-8.
16. Dahiya AV, McDonnell C, DeLucia E, Scarpa A. A systematic review of remote telehealth assessments for early signs of autism spectrum disorder: Video and mobile applications. Pract Innov (Wash D C) 2020;5(2):150-64. doi: 10.1037/ pri0000121.

17. Bent CA, Barbaro J, Dissanayake C. Parents' experiences of the service pathway to an autism diagnosis for their child: What predicts an early diagnosis in Australia? Res Dev Disabil 2020;103:103689. doi: 10.1016/j.ridd.2020.103689.

18. Batool SS, Khurshid S. Factors associated with stress among parents of children with autism. J Coll Physicians Surg Pak 2015;25(10):752-56. doi: 10.2015/JCPSP.752756

19. Barnard-Brak L, Richman D, Ellerbeck K Moreno R. Health care provider responses to initial parental reports of autism spectrum disorder symptoms: Results from a nationally representative sample. Child and Adolesc Ment Health 2017;22(1):30-35. doi: 10.1111/camh.12194.

20. Gibbs V, Aldridge F, Sburlati E, Chandler F, Smith K, Cheng L. Missed opportunities: An investigation of pathways to autism diagnosis in Australia. Res Autism Spectr Disord 2019;57:55-62. doi: 10.1016/j.rasd.2018.10.007.

21. Whitehouse A, Evans K, Eapen V, Wray J. A national guideline for the assessment and diagnosis of autism spectrum disorder in Australia. Brisbane, Qld: Cooperative Research Centre for Living with Autism (Autism CRC), 2018.

22. Gardener H, Spiegelman D, Buka SL. Prenatal risk factors for autism: Comprehensive meta-analysis. Br J Psychiatry 2009;195(1):7-14. doi: 10.1192/bjp. bp.108.051672.

23. Ceylan AC, Citli S, Erdem HB, Sahin I, Acar Arslan E, Erdogan M. Importance and usage of chromosomal microarray analysis in diagnosing intellectual disability, global developmental delay, and autism; and discovering new loci for these disorders. Mol Cytogenet 2018;11:54. doi: 10.1186/ s13039-018-0402-4

24. Miller DT, Adam MP, Aradhya S, et al. Consensus statement: Chromosomal microarray is a first-tier clinical diagnostic test for individuals with developmental disabilities or congenital anomalies. Am J Hum Genet 2010;86(5):749-64. doi: 10.1016/j.ajhg.2010.04.006.

25. Rylaarsdam L, Guemez-Gamboa A. Genetic causes and modifiers of autism spectrum disorder. Front Cell Neurosci 2019;13:385. doi: 10.3389/ fncel.2019.00385.

26. Hiscock H, O Loughlin R, Pelly R, et al. Strengthening care for children: Pilot of an integrated general practitioner-paediatrician model of primary care in Victoria, Australia. Aust Health Rev 2020;44(4):569-75. doi: 10.1071/ AH19177.

27. Milbourn $\mathrm{B}$, Falkmer $\mathrm{M}$, Black $\mathrm{MH}$, et al. An exploration of the experience of parents with children with autism spectrum disorder after diagnosis and intervention. Scand J Child Adolesc Psychiatr Psychol 2017:5(3):104-10. doi: 10.21307/ sjcapp-2017-014.

28. Boshoff K Gibbs D, Phillips RL, Wiles L, Porter L. A meta-synthesis of how parents of children with autism describe their experience of advocating for their children during the process of diagnosis. Health Soc Care Community 2019;27(4):E143-E57. doi: 10.1111/hsc.12691.

29. Catalano D, Holloway L, Mpofu E. Mental health interventions for parent carers of children with autistic spectrum disorder: Practice guidelines from a critical interpretive synthesis (CIS) systematic review. Int J Environ Res Public Health 2018;15(2):341. doi: 10.3390/ijerph15020341.
30. Rattaz C, Alcaraz-Darrou C, Baghdadli A. Benefits of a parental program on stress and quality of life after a diagnosis of autism spectrum disorder (ASD) in their child. Ann Med-Psychol 2016;174(8):644-50. doi:10.1016/j.amp.2015.10.023. 